\title{
Dependence of Oxidation on the Surface Orientation of Tungsten Grains
}

\author{
Karsten Schlueter ${ }^{1,2}$, Martin Balden ${ }^{1}$ \\ ${ }^{1}$ Max-Planck-Institut für Plasmaphysik, Boltzmannstr. 2, D-85748 Garching, Germany \\ ${ }^{2}$ Fakultät für Maschinenwesen, Technische Universität München, D-85748, Garching, Germany
}

\begin{abstract}
The surface orientation of crystals has an influence on various material properties, like the oxidation. A method was developed to correlate the oxidation rate for individual single crystal grains with their surface orientation. The surface orientation of many grains of polycrystalline, recrystallized tungsten samples was determined using electron backscatter diffraction. Subsequently, the samples were oxidized in a thermobalance, measuring the time dependent weight increase. The grain dependent oxidation rates were determined by measuring the thickness of the oxide layer for many individual grains with confocal laser scanning microscopy and scanning electron microscopy on focus ion beam prepared cross-sections. In the temperature range of $720 \mathrm{~K}$ to $870 \mathrm{~K}$, tungsten grains with $\{100\}$ surface orientation have the highest oxidation rate, which is two times higher than the lowest oxidation rate. The lowest oxidation rate belongs to the $\{111\}$ surfaces orientation, while the $\{110\}$ surfaces orientation has an intermediated rate. The derived oxidation rates are consistent with gravimetric measurements. They follow an Arrhenius behavior with an activation energy of 190 $\mathrm{kJ} / \mathrm{mol}$.
\end{abstract}




\section{Introduction}

The crystal orientation has an impact on some surface properties of a material, like irradiation resistance [1], blistering [2], and oxidation [3].The sputter yield differs with the crystal orientation easily by more than a factor of 10 [4]. If a property strongly varies with the crystal orientation, the distribution of the crystal surface orientations, i.e., the texture of materials affects the overall value for that property. Therefore, for comparing results from different studies, the texture of the used materials must be taken into account. On the other hand, by tailoring the texture the overall behavior in an application can probably be optimized. Therefore, a method is developed to determine the crystal orientation dependence of a property; and this method is applied on oxidation of tungsten (W).

The dependence on crystal orientation can be studied on single crystals or on polycrystalline samples. Using single crystals lead to very reliable data, but one experiment for each orientation is required. Therefore, studies on single crystals are time and effort consuming, expensive, and the extrapolation of the behavior to a textured sample can hardly be achieved. Conversely, the main advantage for using polycrystalline samples is that many different crystal orientations are present on one sample and all orientations exhibit the same conditions in the experiment, i.e., variations between different experimental runs are excluded.

The focus in this study lays on the oxidation of tungsten. Many research fields are interested in the use of tungsten oxides [5], while for some applications of $W$ at elevated temperature, oxidation needs to be avoided [6]. Especially in the field of semiconductors e.g. for solar cells [7][8], photocatalysis [9], water splitting [10], sensors [11], and electrochromic devices [12] tungsten oxides are of interest. For example, an application with negative implications of $\mathrm{W}$ oxidations is the use of $\mathrm{W}$ in future nuclear fusion reactors. In case of a loss of coolant with simultaneous air ingress, tungsten can be oxidized [6,13]. In such an incident [14], activated tungsten oxide could be formed and sublimated leading to a potential safety issue if the volatile tungsten oxide is released into the atmosphere. The production of volatile $\mathrm{W}$ oxides could be strongly suppressed by the use of selfpassivating tungsten alloys $[13,15]$.

The oxidation process is complex because it depends on the absorption and diffusion of oxygen in the oxide layer and into the bulk material, the diffusion of tungsten, and the formation of tungsten oxide. For a deeper understanding, dedicated investigations are necessary. Due to the lower density of tungsten oxide compared to tungsten and the difference in the oxidation rates of different grains, a surface roughness on previous flat surfaces will be formed and open-up the possibility to extract the different oxidation rates. In this study a strategy for determining the oxidation rate for many individual grains of previously determined crystal orientation is developed and applied. 


\section{Experimental}

\subsection{Experimental strategy}

Crystal orientation maps of polycrystalline tungsten samples with well-prepared surfaces were obtained by electron backscattering diffraction (EBSD). Thereafter, the samples were oxidized in a thermobalance to get the temporal evolution of the averaged oxidation rates over all grains. Subsequently the absolute thickness of oxide layer was determined for a limited number of grains by scanning electron microscopy (SEM) on focused ion beam (FIB) prepared cross-sections. The three dimensional surface profile data obtained by confocal scanning laser microscope (CLSM) were converted to thickness of the oxide layer for the area pre-analyzed by EBSD. These two microscopic data were overlaid and the oxidation rate was determined for grains with surface misorientation below $10^{\circ}$ to the low indexed planes, i.e. $\{100\},\{110\},\{111\}$.

\subsection{Material and sample preparation}

In order to determine the thickness of the oxide layer on individual grains of known surface orientation, tungsten samples with large enough grains and flat surface are needed. The grains must be large enough to ensure that the grain boundaries do not influence the oxide layer thickness and a constant thickness of the oxide layer is achieved over most of the grain area. Therefore, pieces of $12 \times 10 \times 0.8 \mathrm{~mm}^{3}$ from hot rolled tungsten of the purity of $99.97 \mathrm{wt} \%$ (Plansee SE, Austria) were recrystallized at $2100 \mathrm{~K}$ for 35 minutes in argon atmosphere leading to grain sizes around $20 \mu \mathrm{m}$ (see Fig. 1). The microstructure of the samples from the same tungsten batch, including the distribution of the grain orientations, and the microstructural evolution due to thermal treatment were studied in detail by Manhard et al. [16]. Thereafter, these samples were ground and electro-polished to achieve flat surfaces with height differences between the grains of less than $50 \mathrm{~nm}$. The flatness was checked by surface profile measurements by a CLSM (Olympus, LEXT OLS4000). Details to the electropolishing procedure, which polishes both sides of the tungsten plates, can be found in [17, 18].

In order to align the microscopic data from different techniques, here EBSD and CLSM, an area of the samples was marked with FIB (see Fig. 1) attached to a SEM (FEI, Helios Nanolab 600). These markers could even be used to correct optical differences or distortions (e.g. drift during EBSD in the SEM) in the data sets of the microscopes.

Before the oxidation, the crystal orientation was determined by EBSD (Oxford/HKI, Nordley II detector, Channel 5 acquisition software) in the SEM. From the orientation data (Fig. 2b), grains were selected with their surface misorientated less than $10^{\circ}$ to low indexed planes (Fig. 2c). The later given data related to $\{100\},\{110\},\{111\},\{210\}$, and $\{211\}$ gained on these selections and are cited as, e.g., $\{100\}$ grains. The pre-characterization using EBSD was the most time consuming step in the experiments.

\subsection{Oxidation}

The oxidations of the samples were performed in a thermobalance (Netzsch, STA 449 F1 Jupiter), measuring the time dependent weight increase. For all oxidation experiments, the same heating rate of $20 \mathrm{~K} / \mathrm{min}$ was applied, and the heating was performed in argon atmosphere. Oxidations at constant temperature between $720 \mathrm{~K}$ and $870 \mathrm{~K}$ were performed at normal pressure. The target temperature was hold for $10 \mathrm{~min}$ before the oxidation was started by switching from argon 
atmosphere to a $20 \%$ oxygen $/ 80 \%$ argon atmosphere. The gas flow was $160 \mathrm{ml} / \mathrm{min}$ at room temperature. This was sufficient to replace the atmosphere in the oven chamber of around $80 \mathrm{ml}$ in less than $20 \mathrm{~s}$ at the target temperature above $720 \mathrm{~K}$. The oxidation time depends on the oxidation temperature and was between $30 \mathrm{~min}$ and $5760 \mathrm{~min}(96 \mathrm{~h})$. The aim was to produce the same thickness of the oxide layer for each temperature. The oxidation was stopped by flushing the chamber with argon. The exchange of the atmosphere in the oven chamber was three times faster than the normal gas flow during the experiment. Afterwards, the chamber of the thermobalance was cooled down with a cooling rate of $10 \mathrm{~K} / \mathrm{min}$.

To cross-check the results from the thermobalance, the samples were weighed before and after inserting them in the thermobalance by an analytical balance (Satorious MC25s).

\section{Data evaluation}

The oxidized samples were evaluated in the following steps:

- Measuring the relative height from an oxidized sample with CLSM

- Assigning the relative height to the crystal orientation by overlaying he CLSM image and the orientation map obtained by EBSD

- Determining the absolute thickness of the oxide layer on single crystal grains with their surface close to the $\{100\}$ orientation by SEM on FIB prepared cross-sections

- Level the relative height on grains with the $\{100\}$ surface orientation and calculating the thickness of the oxide layer for the other surface orientations

EBSD data and the relative height data from the CLSM were merged manually by overlaying the images. For this aim a graphic tool called GIMP was used. An example of an overlay is shown in figure 2d.

EBSD images (figures 2(b,c)) and a CLSM image (figure 2(a)) were overlaid (figure 2(d)) to assign the height to the grain orientation. The relative height for each grain was extracted by the measuring software of the CLSM. With the SEM, the absolute values of the oxide layer thickness were measured on FIB prepared cross-sections. A platinum carbon layer was deposit to cover the oxide layer for getting accurate height measurements of the oxide layer. The FIB cut was done with Ga ions at $30 \mathrm{keV}$. At least eight cross-sections on each sample were prepared on crystal grains with $\{100\}$ surface orientation. This surface orientation has the highest oxidation rate and, therefore, the thickest oxide layer.

The relative heights from the CLSM were used to calculate the absolute height of the grains with surface orientation $\{110\}$ and $\{111\}$. From the number density of tungsten $\left(6.32 \mathrm{e} 22 \mathrm{~W} / \mathrm{cm}^{3}\right)$ and tungsten oxide $\left(1.95 \mathrm{e} 22 \mathrm{~W} / \mathrm{cm}^{3}\right)$ and the assumption of expansion of the oxide layer only in one direction, it is expected that the $70 \%$ of the oxide layer is elevated above the original surface level, while $30 \%$ of it lays below the original surface. The theoretical quotient $f_{c t}$ of the part of the layer below ( $h_{\text {below }}$ ) to above $\left(h_{\text {above }}\right)$ the original surface is 0.42 and can be used to obtain absolute thicknesses. This value will be higher if the oxide layer expands in more than one direction and lower if the oxide layer is not dense, i.e., has pores and cracks. The growth of tungsten oxide below the original surface was verified on cross-sections. At grain boundaries of grains with different oxidation rates, it is possible to measure height differences between the oxide layers, separated in the part above and below the original surface. An example is shown in figure 3. Both, hrel $\{h k /\}$ and $h_{\text {depth }\{h k l\}}$ were measured and the quotient $f_{c e}$ was calculated as

$$
f_{c e}=h_{\text {below }} / h_{\text {above }}=h_{\text {depth }\{h k l\}} / h_{\text {rel }\{h k l\}} .
$$


Overall, 22 measurements were performed at grain boundaries selected regarding height difference and not regarding the orientation of the neighboring grains. The mean measured ratio $f_{c e}$ is $0.45 \pm 0.13$ between depth growth and height growth. The error of 0.13 is the standard deviation of the measurements. The experimentally determined value ratio $f_{c e}$ is very close to the theoretical one $\left(f_{c t}\right)$ of 0.42 .

Finally, the absolute thickness of the oxide layer for different crystal orientations could be calculated using equation 2.

$$
h_{a b s}\{\mathrm{hkl}\}=h_{a b s}\{100\}-h_{\text {rel }\{\mathrm{hkl}\}}(1+0.45)
$$

where $h_{a b s[100\}}$ is the absolute height determined by SEM on FIB prepared cross-sections, $h_{\text {rel\{hkl\} }}$ is the relative value of the $\{\mathrm{hkl}$ \} grain obtained by the CLSM and the last term is the correction for the depth growth using the experimental determined value for the quotient $f_{c}$. Height information of the CLSM and thickness measurement on grains with the $\{100\}$ surface orientation by SEM are necessary to obtain the oxide thickness for the complete analyzed area. The $\{100\}$ surface orientation has the thickest oxide layer and this orientations was therefore chosen to achieve accurate thickness measurement. This procedure allows to evaluate thickness measurements on many different single crystal grains with selected orientation at one sample.

\section{Results}

\subsection{Oxidation in respect to the surface orientation}

The main task is the determination of the thickness of the oxide layer on each grain. First, measurements obtained by the CLSM were transformed using equation 2 into thickness data. The height data of the central area on each grain were averaged. The non-uniform oxide layer close to the grain boundaries was excluded for the thickness measurement. To cross-check, the thickness was determined by a second measurement method: Several cross-sections were prepared and imaged on each sample to obtain an average thickness value of each low index crystal orientation. The grains for the cross-sections were selected regarding their crystal orientations, known from the EBSD data.

As the samples exhibit a strong texture [16], i.e., some crystal orientations dominate the sample surface, more grains with surfaces close to $\{100\}$ orientation and $\{110\}$ orientation are present on the sample than with the $\{111\}$ orientation. Therefore, more measurements were performed on the $\{100\}$ and $\{110\}$ orientation than on the $\{111\}$ orientation. As a consistent check of the described thickness determination (eq. 2), the CLSM data was compared to thickness measurement on FIB prepared cross-sections using SEM for other orientation than the $\{100\}$ surface orientation. At least 10 measurements were done on FIB prepared cross-sections and the standard deviation is taken as error. With the CLSM much more values are determined (between 46-225 measurements as shown in figure 4) and the standard deviation is also taken as error. The calculated mean values from the CLSM are in the error range of the thickness measurements on FIB prepared cross-sections obtained by SEM. The $\{210\}$ and $\{211\}$ orientation were also evaluated to have two additional crystal orientations to compare the developed method with CLSM to the FIB prepared cross-sections. The results are shown in figure 4.

The results were also compared with gravimetrical analyses. To compare the thickness measurement from the newly developed method with oxidation rates derived from weight gain measured with the analytical balance and thermobalance, the thickness measurements are converted into parabolic oxidation rates in $\mathrm{mg}^{2} /\left(\mathrm{cm}^{4 *} \mathrm{~s}\right)$ according to 


$$
K_{p}=\frac{\left(L_{\text {thickness }} * \rho_{W O_{3}} * \frac{3 * u_{O}}{u_{W}+3 * u_{O}}\right)^{2}}{t_{o x}},
$$

where $K_{p}$ is the parabolic oxidation rate, Lthickness is the thickness of the oxide layer, $\rho_{\text {wo }}=7.25 \mathrm{~g} / \mathrm{cm}^{3}$ [19] is the density of tungsten oxide, tox is the oxidation time and $3 u_{0} /\left(u_{w}+3 u_{0}\right)$ is the ratio of the atomic mass of oxygen and tungsten oxide. The reason for using the atomic mass ratio is that the thermo balance and analytical balance measured the mass increase by oxygen uptake and not the mass of tungsten oxide. It is unknown which tungsten oxides are formed during the oxidation. But from literature it is expected to be $\mathrm{WO}_{3}$ and $\mathrm{WO}_{2.75}$ for temperatures between $500 \mathrm{k}$ and $900 \mathrm{~K}$ under normal pressure [3] [19]. Fortunately, the product of density and the atomic mass ratio is similar for all tungsten oxides as shown in table 1. The error is less than 10 percent.

In figure 5 the comparison of the parabolic oxidation rates is presented. The oxidation rates of the balances are between the rates for surfaces close to $\{111\}$ and $\{100\}$ obtained from the thickness measurements. This is expected, because the balances give a mean value over all orientations.

Overall, the developed method yields consistent results and the grains with the $\{100\}$ surface orientation have the highest oxidation rate, which is two times higher than those with the lowest oxidation rate. The grains with $\{111\}$ surface orientation belong to those with lowest oxidation rate, while the $\{110\}$ surface orientation has an intermediated rate.

\subsection{Temperature dependent oxidation in respect to the surface orientation}

The developed method was applied to investigate grain dependent oxidation at different temperatures. To facilitate the comparison at different temperatures, it was aimed at having the same thickness of the oxide layer. Therefore, the oxidation times were adjusted by taking into account oxidation rates from literature [20] (fig. 7).

Five independent oxidations were performed at a temperature of $870 \mathrm{~K}$ because the method development was performed at this temperature. For the other oxidation temperatures only one sample was oxidized. But a large area were measured with EBSD and at least 15 values were measured in the $\{111\}$ orientation and fare more in the other crystal orientations. The standard derivation was taken as error of the measurements. The grain dependent oxidation at different temperatures is shown in figure 6. The absolute thickness of the oxide layer is plotted against the grain orientation. The grain specific evaluation shows the same orientation dependency for all temperatures, implying the same oxidation behavior for the oxide layer growth up to several micrometers.

The thickness measurements were converted into parabolic oxidation rates with equation 3 to compare the data with the data obtained from gravimetric measurements and the data of Koch et al. [20]. The comparison is shown in figure 7. It is an Arrhenius plot which describes the dependence of a chemical reaction rate on the inverse absolute temperature.

Koch et al. [20] oxidized from $873 \mathrm{~K}$ to $1273 \mathrm{~K}$. The oxidations in this work were performed for $30 \mathrm{~min}$ at $870 \mathrm{~K}$ whereas Koch et al. [20] oxidized for $12 \mathrm{~h}$, i.e., achieving much thicker oxide layers. During a longer oxidation the oxide layer is influenced by cracks and a linear oxidation rate would be expected. Therefore, the parabolic oxidation rates of pure tungsten from [20] can be slightly too high. Nevertheless, the regression is done with the "thermobalance" data and the oxidation rate of [20]. The activation energy is $190 \mathrm{~kJ} / \mathrm{mol}$. The parabolic oxidation rates from the thermobalance are given in table 2. 


\section{Discussion}

Oxidation is a complex process. The oxidation of tungsten depends on several parameters like roughness of the surface, the absorption of oxygen, the diffusion of tungsten and the diffusion of oxygen.

For the analyzed samples, the roughness has an influence at the very beginning of the oxidation. Therefore, the samples were prepared with a roughness in the order of some $\mathrm{nm}$. The tungsten oxide expands around $70 \%$ above and $30 \%$ below the original surface level. An oxide layer with a thickness around $4 \mu \mathrm{m}$ means that tungsten oxidizes around $1 \mu \mathrm{m}$ below the original tungsten surface. Therefore, a tungsten surface with a roughness of some $\mathrm{nm}$ influences the oxidation only for a short time until the nm deep scratches are oxidized.

At the starting point of the oxidation, oxygen is absorbed at the tungsten surface. Engel and Gomer [21] measured the absorption of inert gases (e.g. argon) into tungsten with different surface orientations. The $\{110\}$ surface orientation has the highest absorption. But the highest oxidation rate on tungsten is found for the $\{100\}$ surface orientation. If oxygen had the same behavior like argon, it would mean that the grain dependent oxidation is not triggerd by the absorption of oxygen.

Another parameter to explain the order of the oxidation rate respective to surface orientation could be the atomic surface density of the low indexed surfaces. Ligenza [22] oxidized silicon with different crystal orientations. It was suggested that the crystal orientation effect depends on the surface density of atoms in the first atomic layer. The argumentation was that the number of bonds is important and the Si-Si bonds had to be broken to proceed the oxidation. Tungsten has a bcc-lattice and the $\{110\}$ surface orientation has the highest surface density of atoms in the first atom layers but has an oxidation rate between the $\{111\}$ and $\{100\}$ surface orientation. Therefore, these argumentation does not fit to the tungsten oxidation either.

There are many effects which influence the oxidation and maybe it is a combination of some of them that leads to the final result. To distinguish these effects there must be a closer look to the different mechanisms on the oxidation.

Oxidation behavior is different over the time, i.e. oxide layer thickness, because different mechanisms are involved and three will be discussed. First, there is no oxidation layer on the tungsten surface. Therefore, oxygen does not need to diffuse at the very beginning of the oxidation through an oxide layer to the tungsten surface. The oxidation is rapid at the very beginning and, therefore the mass increases rapidly. After the first oxide layers are formed, oxygen must diffuse through an oxide layer. A parabolic behavior of the oxidation rate is expected for this phase. After a certain oxide thickness is reached, cracks influence the oxidation and change the parabolic oxidation behavior into a linear oxidation behavior. To learn more about the oxidation dependence on different crystal orientations, the next step is to distinguish these three mechanisms by evaluating the crystal dependency for thinner and thicker oxide layers. Oxide layer on the nm scale could show surface effects, i.e. exclude diffusion processes in the oxide layer, and thicker oxide layer could show influences of diffusion or cracks. First measurements give a hint that the crystal dependency changes for different thicknesses of the oxide layer. 


\section{Summary}

A method was developed to measure the thickness of oxide layers for individual grains with low indexed surface. On the basis of image overlays, the data from two different measurement systems were merged together. The height data were obtained by a confocal laser scanning microscope (CLSM), and the grain orientation data were obtained by the electron backscatter diffraction (EBSD) measurements. On a tungsten surface the tungsten oxide expands around $70 \%$ above and $30 \%$ below the original surface level. The three dimensional surface data of the CLSM were transformed into absolute oxide layer thickness using the thickness measurements on focused ion beam (FIB) prepared cross-sections imaged by scanning electron microscopy (SEM).

The absolute thickness of the oxide layer varies by a factor of two between the $\{100\}$ and $\{111\}$ orientation. The $\{110\}$ orientation has an oxidation rate between the $\{100\}$ and $\{111\}$ orientation. It was observed that other high index orientations have a low oxidation rate like the $\{111\}$ surface orientation.

The thickness measurements were compared with results obtained by gravimetric analyses. The mass gain due to oxidation, measured by thermobalance and analytical balance, were transformed into parabolic oxidation rates in $\mathrm{mg}^{2} / \mathrm{cm}^{4} \cdot \mathrm{s}$. Also the thickness data are transformed according to equation 3 to parabolic oxidation rates. The rates obtained from the different techniques agree well.

The developed method was applied to study the temperature dependence of the oxidation rate of tungsten in the temperature range $720 \mathrm{~K}$ to $870 \mathrm{~K}$ for the low indexed surfaces. This temperature dependence is well described by an activation energy of $190 \mathrm{~kJ} / \mathrm{mol}$.

The introduced method will be extended by a semi-automated program, which allows to analyze the grain dependency for many surface orientations. In addition, oxidation experiments are planned to distinguish different oxidation phases, e.g., by studying different thicknesses of the oxide layer. This could give a hint if the crystal dependent oxidation is dominated by the oxide layer or by other influences, e.g. the surface binding energy of tungsten atoms. 


\section{References}

[1] Garrison, L. M., \& Kulcinski, G. L. Irradiation resistance of grains near $\left\{\begin{array}{lll}0 & 1\end{array}\right\}$ on polycrystalline tungsten under $30 \mathrm{keV} \mathrm{He}+$ bombardment at 1173 K. Physica Scripta, 2014; (T159), 014020.

[2] Jia, Y. Z., De Temmerman, G., Luo, G. N., Xu, H. Y., Li, C., Fu, B. Q., \& Liu, W. Surface morphology and deuterium retention in tungsten exposed to high flux $\mathrm{D}$ plasma at high temperatures. Journal of Nuclear Materials, 2015; 457, 213-219.

[3] Alimov, V. K., Tyburska, B., Balden, M., Lindig, S., Roth, J., Isobe, K., \& Yamanishi, T. Surface morphology and deuterium retention in tungsten oxide layers exposed to low-energy, high flux D plasma. Journal of Nuclear Materials, 2011; 409(1), 27-32.

[4] Roosendaal, H. E. Sputtering yields of single crystalline targets. In Sputtering by particle bombardment I (pp. 219-256). Springer, Berlin, Heidelberg, 1981;

[5] Bignozzi, C. A., Caramori, S., Cristino, V., Argazzi, R., Meda, L., \& Tacca, A. Nanostructured photoelectrodes based on $\mathrm{WO}_{3}$ : applications to photooxidation of aqueous electrolytes. Chemical Society Reviews, 2013; 42(6), 2228-2246.

[6] Smolik, G. R., Piet, S. J., \& Neilson Jr, R. M. Predictions of Radioactive Tungsten Release for Hypothetical ITER Accidents. Fusion Technology, 1991; 19(3P2B), 1398-1402.

[7] Varghese, O. K., Paulose, M., \& Grimes, C. A. Long vertically aligned titania nanotubes on transparent conducting oxide for highly efficient solar cells. Nature nanotechnology, 2009; 4(9), 592597.

[8] Hara, K., Zhao, Z. G., Cui, Y., Miyauchi, M., Miyashita, M., \& Mori, S. Nanocrystalline electrodes based on nanoporous-walled $\mathrm{WO}_{3}$ nanotubes for organic-dye-sensitized solar cells. Langmuir, 2011; 27(20), 12730-12736.

[9]Yu, J., \& Qi, L. Template-free fabrication of hierarchically flower-like tungsten trioxide assemblies with enhanced visible-light-driven photocatalytic activity. Journal of hazardous materials, 2009; 169(1), 221-227.

[10]Lai, C. W., \& Sreekantan, S. Incorporation of $\mathrm{WO}_{3}$ species into $\mathrm{TiO}_{2}$ nanotubes via wet impregnation and their water-splitting performance. Electrochimica acta, 2013; 87, 294-302.

[11] Wang, X., Miura, N., \& Yamazoe, N. Study of $\mathrm{WO}_{3}$-based sensing materials for $\mathrm{NH}_{3}$ and $\mathrm{NO}$ detection. Sensors and Actuators B: Chemical, 2000; 66(1), 74-76.

[12] Berger, S., Tsuchiya, H., Ghicov, A., \& Schmuki, P. High photocurrent conversion efficiency in selforganized porous $\mathrm{WO}_{3}$. Applied Physics Letters, 2006; 88(20), 203119.

[13] Koch, F., \& Bolt, H. Self passivating W-based alloys as plasma facing material for nuclear fusion. Physica Scripta, 2007; (T128), 100.

[14] Maisonnier, D., Cook, I., Pierre, S., Lorenzo, B., Edgar, B., Karin, B., ... \& Prachai, N. The European power plant conceptual study. Fusion Engineering and Design, 2005; 75, 1173-1179. 
[15] Calvo, A., García-Rosales, C., Ordás, N., Iturriza, I., Schlueter, K., Koch, F., ... \& Pastor, J. Y. Selfpassivating W-Cr-Y alloys: characterization and testing. Fusion Engineering and Design. 2017; 124, 1118-1121.

[16] A Manhard, M Balden, \& S Elgeti. Quantitative microstructure and defect density analysis of polycrystalline tungsten reference samples after different heat treatments. Practical Metallography Praktische Metallographie, 2015; 52:437- 466.

[17] Manhard, A., Matern, G., \& Balden, M. A step-by-step analysis of the polishing process for tungsten specimens. Practical Metallography, 2013; 50(1), 5-16.

[18] K. Schlueter IPP-Report, Masterarbeit http://hdl.handle.net/11858/00-001M-0000-002C-94A5-8

[19] E Lassner and W D Schubert. Tungsten: Properties, Chemistry, Technology of the Elements, Alloys, and Chemical Compounds. Springer US, 1999. ISBN 9780306450532.

[20] Koch, F., Brinkmann, J., Lindig, S., Mishra, T. P., \& Linsmeier, C. Oxidation behaviour of siliconfree tungsten alloys for use as the first wall material. Physica Scripta, 2011; (T145), 014019.

[21] Engel, T., \& Gomer, R. Adsorption of inert gases on tungsten: measurements on single crystal planes. The Journal of Chemical Physics, 1970; 52(11), 5572-5580.

[22] Ligenza, J. R. Effect of crystal orientation on oxidation rates of silicon in high pressure steam. The Journal of Physical Chemistry, 1961; 65(11), 2011-2014. 
Table 1: Densities of different tungsten oxides [19]. The values in the column "Density* Atomic atomic mass ratio" vary by less than $10 \%$.

\begin{tabular}{c|c|c|c}
$\begin{array}{c}\text { Tungste } \\
\text { n Oxide }\end{array}$ & Density & Atomic mass ratio & $\begin{array}{c}\text { Density* atomic mass } \\
\text { ratio }\end{array}$ \\
\hline $\mathrm{WO}_{3}$ & 7.25 & 0.20 & 1.50 \\
\hline $\mathrm{WO}_{2} .9$ & 7.8 & 0.19 & 1.49 \\
\hline $\mathrm{WO} 2$ & 10.9 & 0.14 & 1.61
\end{tabular}

Table 2: Oxidation rates at different temperatures. The oxidation rates for the temperatures at $873 \mathrm{~K}, 1073 \mathrm{~K}$ and $1273 \mathrm{~K}$ are taken from Koch et al. [20].

\begin{tabular}{c|c|c} 
Temperature / K & Time / & $\mathrm{Kp} / \mathrm{mg}^{2} /\left(\mathrm{cm}^{4 *} \mathrm{~s}\right)$ \\
\hline 720 & 96 & $4.3 \times 10^{-7}$ \\
\hline 820 & 2.5 & $1.3 \times 10^{-5}$ \\
\hline 870 & 0.5 & $6.3 \times 10^{-5}$ \\
\hline 873 & 12 & $1.8 \times 10^{-4}$ \\
\hline 1073 & 12 & $8.0 \times 10^{-3}$ \\
\hline 1273 & 12 & $4.5 \times 10^{-1}$
\end{tabular}




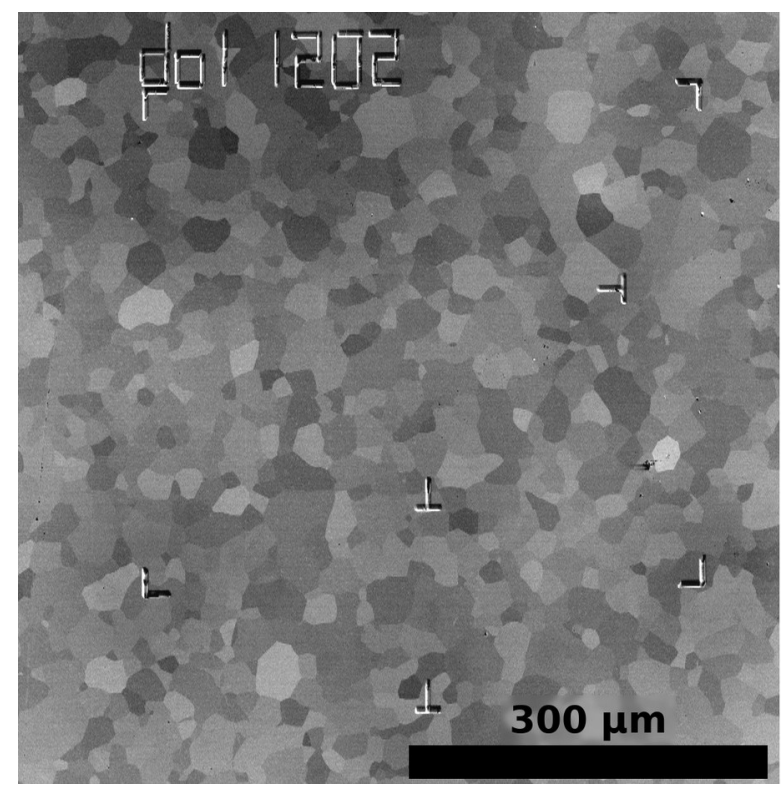

Figure 1: SEM image showing four $T$ markers and four $L$ markers on a tungsten sample used for aligning the EBSD and CLSM data. In addition to these markers, the sample name is labeled with FIB.

a)

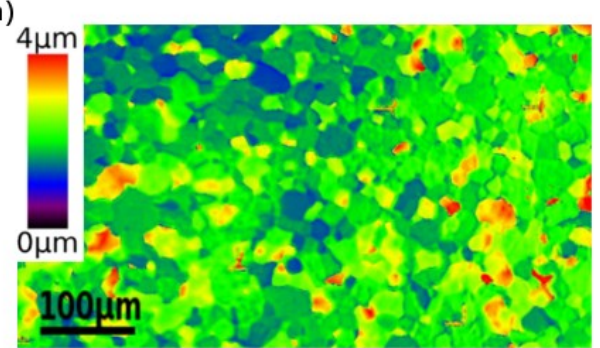

c)

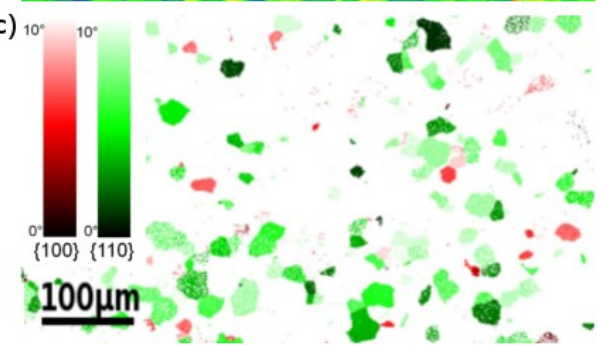

b)

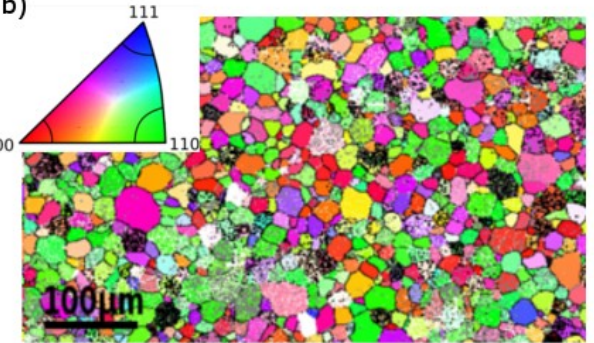

d)

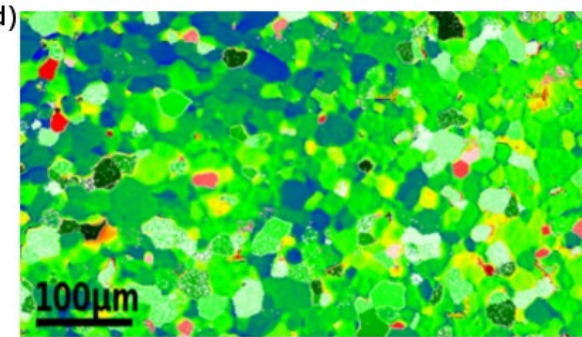

Figure 2: An example of merging the data: (a) height profile image from the CLSM; (b) orientation map obtained by EBSD; (c) selection of grains with misorientation of less than $10^{\circ}$ to low index surfaces; (d) overlay of (a) and (c). 
a)

b)

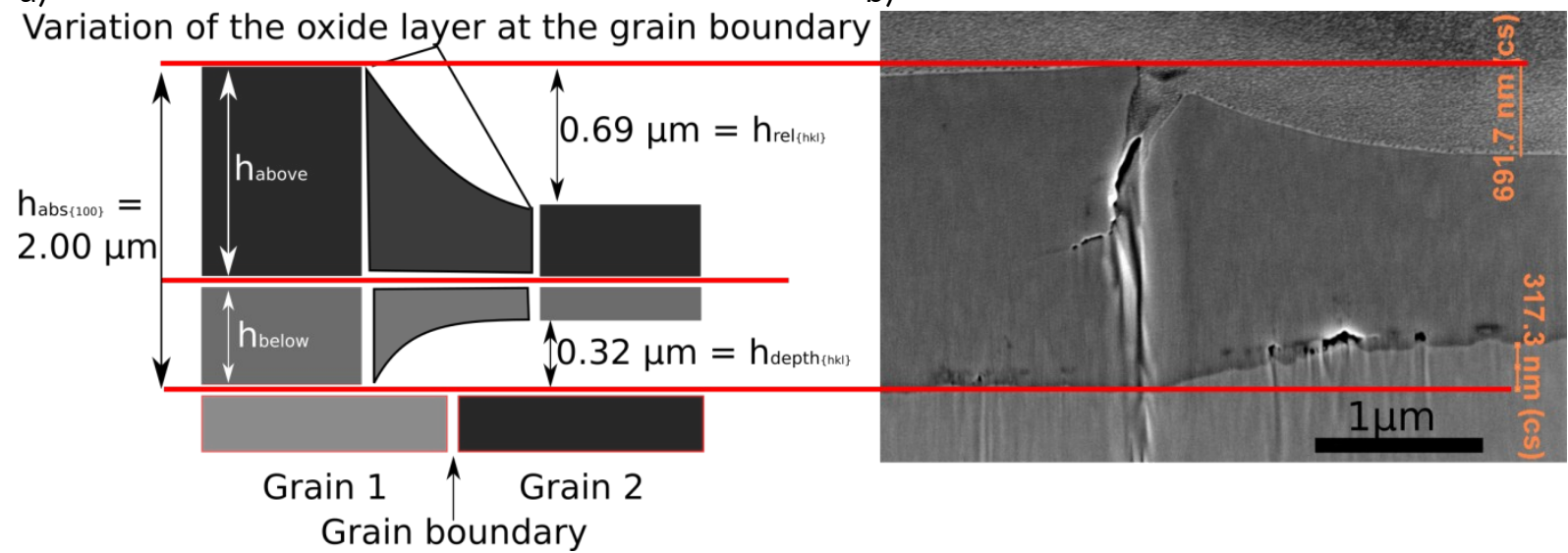

Figure 3: (a) Illustration of the experimental measurement of the correction factor to convert the CLSM data to absolute thickness data; (b) SEM image of cross-section through oxide layer. In the example, grain 1 has roughly $\{110\}$ orientation with a high oxidation rate and grain 2 roughly $\{111\}$ orientation with a low oxidation rate.

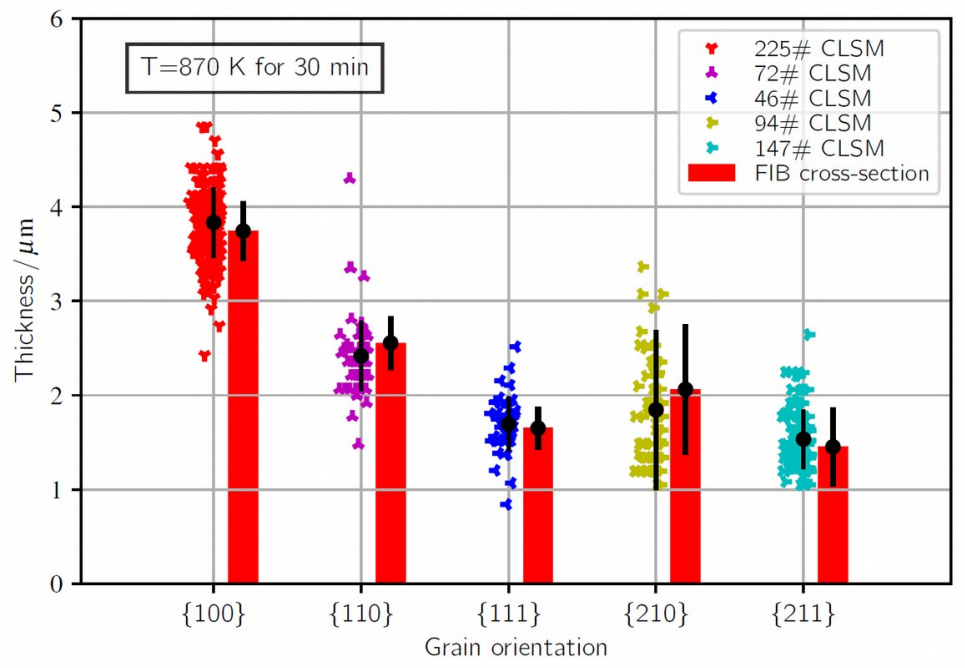

Figure 4: Grain dependent oxidation at $870 \mathrm{~K}$ for $30 \mathrm{~min}$. The thickness of the oxide layer is plotted against the surface orientation of grains misorientated less than $10^{\circ}$ to five $\{\mathrm{hkl}\}$ planes. The calculated values from the CLSM measurements are shown as points. The absolute thickness values obtained on FIB-prepared cross-sections are shown as bars to compare them with the calculated values from the CLSM. This figure includes data from samples of five independent incubations and the "\#" symbol stands for the number of measurements. The mean value with their standard deviation as error bars are plotted, too. 


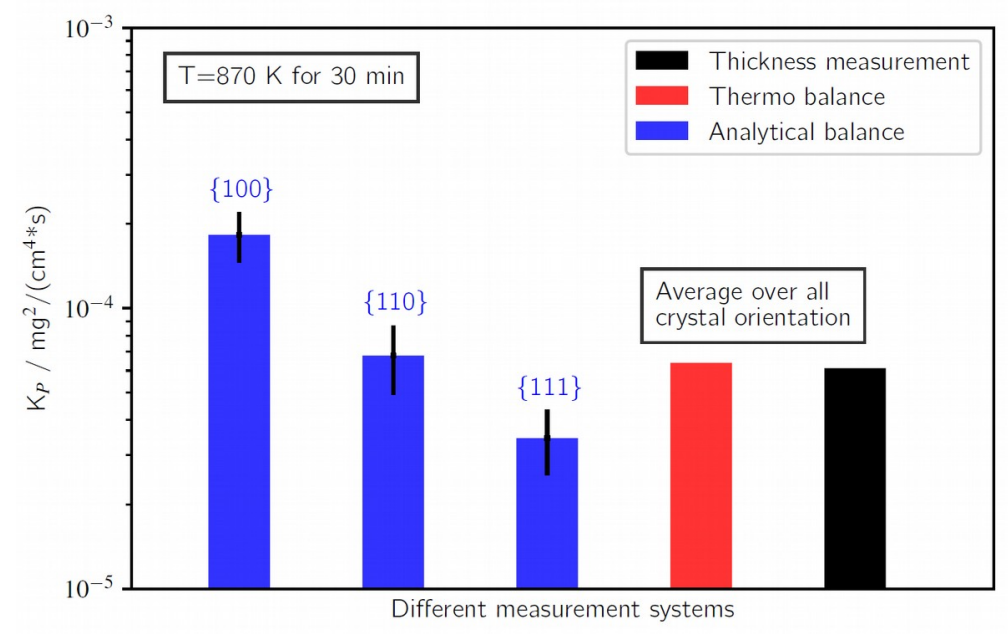

Figure 5: The parabolic oxidation rates $\left(K_{p}\right)$ obtained by different methods. The data of the thermobalance, analytical balance and the thickness measurement are mean values of at least five independent oxidation experiments. The surfaces close to $\{100\}$ have the fastest oxidation and those close to $\{111\}$ have one of the slowest. The oxidations were performed at $870 \mathrm{~K}$ for 30 minutes.

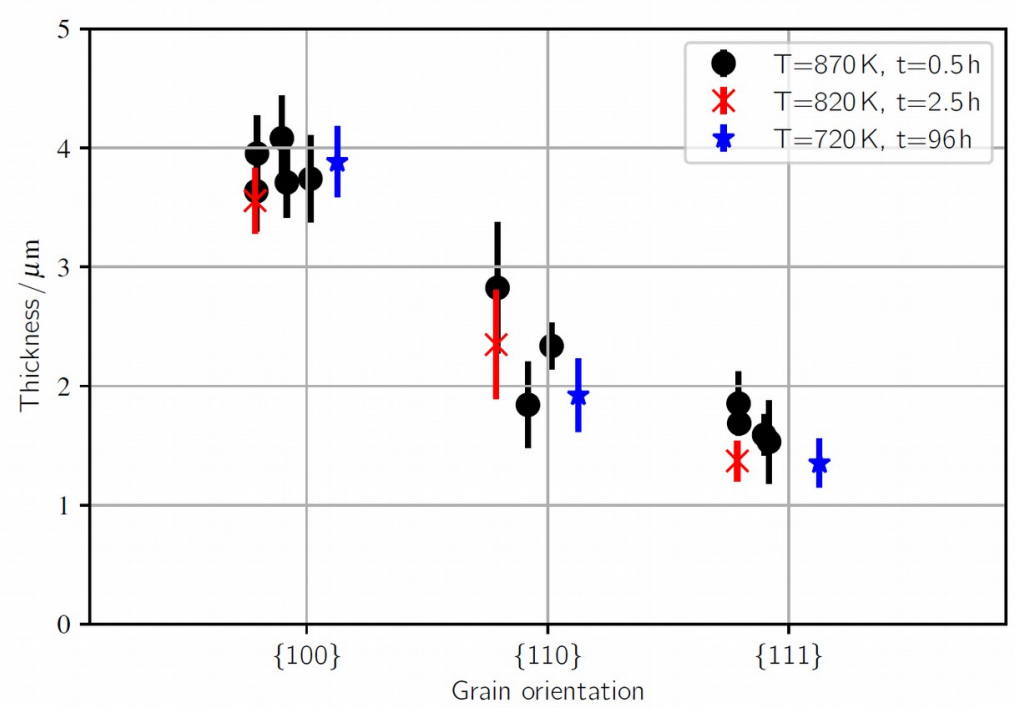

Figure 6: Grain dependent oxidation at three different temperatures for surface orientations misorientated less than $10^{\circ}$ to low indexed planes. The absolute thickness of the oxide layer is plotted against the grain orientation. Due to the strong texture and the restricted area of the EBSD measurement, at least one of the five samples could not be evaluated for the $\{110\}$ and \{111\} orientation [16]. 


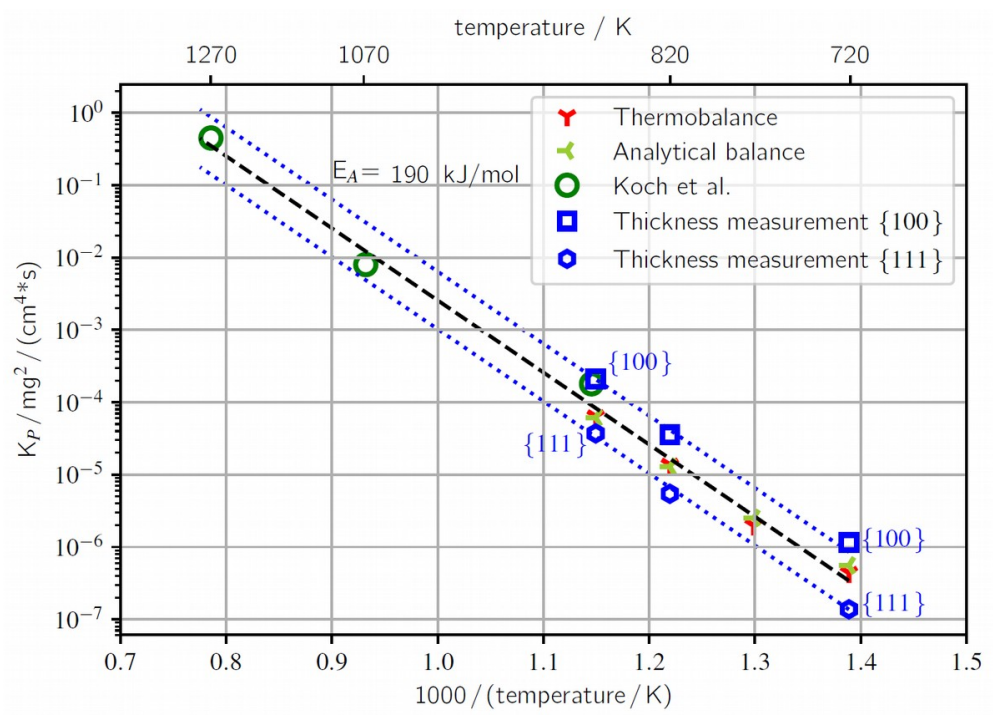

Figure 7: The parabolic oxidation rate is plotted against the inverse of the temperature. This figure compares data of different measurement systems with the data from Koch et al. [20]. The "thermobalance" data, including the data of Koch et al. [20], are used for the regression. The black dashed line shows a linear regression with the same relative errors for each data point. The blue dotted lines are shifted lines to show that the activation energy is equal for each crystal orientation. The activation energy is $E_{A}=190$ $\mathrm{kJ} / \mathrm{mol}$. 